\title{
Cerebral Venous Thrombosis in Evan's Syndrome: An Unusual Presentation
}

\author{
Cheng MH, Norzila TAB \\ Department of Radiology, School of Medical Sciences, Universiti Sains Malaysia
}

\begin{abstract}
Cerebral Venous Thrombosis in patients with Evan's Syndrome of autoimmune hemolytic anemia is rare. The common neurological symptoms are headaches, vision loss, dyslexia without agraphia, motor aphasia, unilateral upper limb weakness and papilloedema. We present a case report of a lady with a known case of Evan's Syndrome whom presented with severe anemia and unilateral right sided hemiparesis with right facial weakness. Plain and Contrast enhanced CT brain showed bilateral high parietal white matter edema with venous thrombosis in the right transverse and superior sagittal venous sinuses. At the time of the diagnosis, she was in hematological remission.
\end{abstract}

Key Words: Evan's Syndrome, Autoimmune Hemolytic Anemia, Cerebral Venous Thrombosis

\section{INTRODUCTION}

Evan's Syndrome of autoimmune hemolytic anemia is a rare hematological disorder. It is named after Dr Robert Evans who described the relationship between acquired hemolytic anemia and primary thrombocytopenic purpura in 1951. The disorder is defined as simultaneous or sequential development of Coombs-positive autoimmune hemolytic anemia (AIHA) and immune thrombocytopenia and/or immune neutropenia in the absence of any underlying cause. ${ }^{3}$

Incidence of cerebral venous thrombosis in Evan's Syndrome is unusual. Only a few cases in the literature described the incidence attributed to the medication. ${ }^{1,5,6}$ Patient usually presents with nonspecific neurological symptoms. In this report, we emphasize the importance of neuroimaging and highlight the radiological pitfalls in diagnosing cerebral venous sinus thrombosis.

\section{CASE REPORT}

A 41-year old Malay lady, who is a known case of Evan Syndrome, presented to HUSM emergency

\footnotetext{
Corresponding author:

Dr Cheng Ming Huan

Department of Radiology,

School of Medical Sciences,

Universiti Sains Malaysia (USM),

16150 Kubang Kerian, Kelantan, Malaysia.

Office : 09-7673468,

HP: $012-4076378^{1} 012-2886284^{2}$

Fax : 09-7673468

Email :cmhuan@hotmail.com
}

department with severe anemia, right sided hemiparesis with right facial weakness. She was diagnosed with the disease at the age of 33 years through positive direct Coombs test. Her serum haemoglobin $(\mathrm{Hb})$ level at the initial presentation was $2.6 \mathrm{~g} / \mathrm{dL}$. She was treated with multiple $\mathrm{Hb}$ transfusion and received oral prednisolone. Her medication dosage was reduced gradually and stopped after 1 year as she achieved haematological remission (serum $\mathrm{Hb}$ level maintained above $8.0 \mathrm{~g} /$ $\mathrm{dL})$.

However, she had one episode of relapse in June 2014 where she required hospitalisation for $\mathrm{Hb}$ transfusion and intravenous methylprednisolone treatment. She was discharged from the hospital with oral prednisolone. At day 4 after hospital discharge, her condition worsened and she came back to emergency department with the above mentioned complaint.

Physical examination showed that she was pale, tachycardic and tachypneic. There was weakness noted in the right hemifacial region, right upper (Grade 3) and lower limbs (Grade 4). The serum $\mathrm{Hb}$ was $6.3 \mathrm{~g} / \mathrm{dL}$.

Clinically there was no evidence of deep venous thrombosis. Oral prednisolone was continued. In view of her neurological symptoms, she underwent contrast enhanced CT brain for further evaluation.

\section{DISCUSSION}

Cerebral venous sinus thrombosis in patients with Evan's Syndrome of autoimmune hemolytic anemia is rare. Only three reports were found in the literature that described these rare findings. 1,5,6 
The first case described the occurrence of superior sagittal sinus thrombosis which was related to administration of therapeutic agents including 6mercaptopurine and high dose of steroids. ${ }^{5}$ The second study reported a patient with Evan syndrome who developed deep vein thrombosis and pulmonary thrombosis after intravenous immunioglobulin (IVIG) therapy.

The authors postulated that IVIG may induce thrombosis by increasing blood viscosity, platelets activation or vessel vasospasm. ${ }^{1}$ The third patient described in the literature was in hematological remission and was on lamivudine for his underlying chronic hepatitis B treatment.

However, no established evidence in the literature to suggest an association between lamivudine treatment and venous sinus thrombosis. ${ }^{6}$ In this case, our patient had history of IV metylprednisolone and was on oral prednisolone during the CT brain. Symptoms of cerebral venous sinus thrombosis are usually non specific. Common symptoms are headache, focal neurological deficits, seizures and altered conscious level. ${ }^{4}$ Therefore cerebral venous thrombosis is easily overlooked clinically as well as radiologically. Imagings include CT and MRI brain plays crucial role in diagnosing cerebral venous thrombosis. On plain CT brain, the classical direct sign of cerebral venous thrombosis is "dense clot" sign.

However, this sign only occurs in 30\% of cases. Another sign called "dense cord" sign describes linear hyperdensity representing thrombosed cortical vein in plain $\mathrm{CT}$ brain. The indirect signs are common but non-specific for cerebral thrombosis on CT brain. Those signs are diffuse brain edema and venous infarction. ${ }^{4}$ The location of infarction/edema on $\mathrm{CT}$ brain is useful in predicting the venous sinuses where thrombosis occurs (Table 1 ).

In our patient, her plain CT brain showed white matter hypodensities in both parietal lobes with "dense clot" sign in the superior sagittal sinus (Figure 1). Contrast enhanced CT image confirmed thrombosis in the venous sinus (Figure 2 ).

\begin{tabular}{cc}
\hline Location of infarction & Expected thrombosis in the venous drainage \\
\hline Sagittal sinus & Parasagittal region \\
Temporal lobe & Labbe's vein \\
Thalami, basal ganglia and internal capsule & Deep venous sinus \\
\hline
\end{tabular}

Table 1. Location of the infarction with respect to the expected course of venous drainage (Poon et al., 2007)
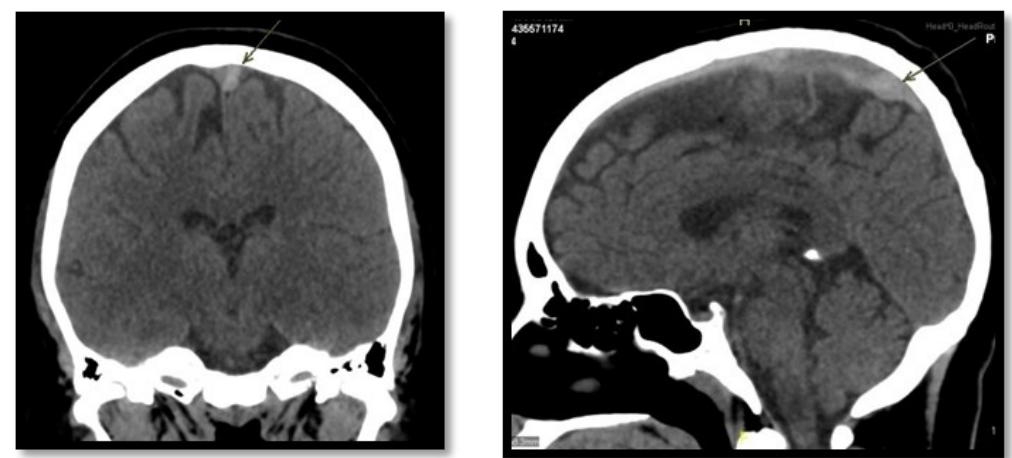

Figure 1. Plain CT brain shows "dense clot" sign, indicating hyperdensity within the superior sagittal sinus secondary to thrombosis. This is a classical direct sign of cerebral venous thrombosis.
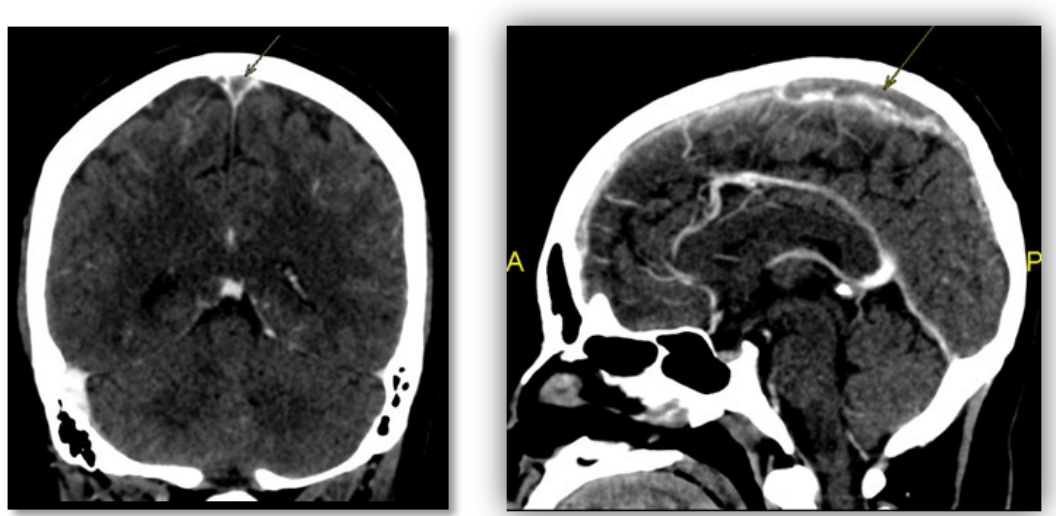

Figure 2. Contrast enhanced CT brain shows filling defect in the right transverse sinus and superior sagittal sinus ("empty delta" sign), suggestive of venous sinus thrombosis. 
On contrast enhanced CT brain, the "empty delta" sign is specific for cerebral venous thrombosis. However, ten to thirty percent of cases may appear normal on plain and contrast enhanced CT brain. In this condition, CT venography or MRI with $M R$ venography is the gold standard of assessment. ${ }^{2}$

Our patient was treated with intravenous heparin therapy during admission and subsequently was on oral Warfarin with close monitoring of INR. On follow up after 4 months, repeated MRI brain showed no evidence of thrombosis. Patient recovered well without any permanent disability in neurology.

\section{CONCLUSION}

Cerebral venous thrombosis associated with Evan's Syndrome of autoimmune hemolytic anemia is very rare. Cerebral venous thrombosis is easily overlooked on imaging. Therefore, radiologists should be aware of normal venous sinuses anatomy and pitfalls during image interpretation in order to make accurate diagnosis. Early diagnosis and treatment can prevent permanent brain parenchyma damage.

\section{REFERENCES}

1. Emerson, G. G., Herndon, C. N. \& Sreih, A. G. (2002). Thrombotic Complications After Intravenous Immunoglobulin Therapy in Two Patients. Pharmacotherapy: The Journal of Human Pharmacology and Drug Therapy, 22(12), 1638-1641. doi: 10.1592/phco.22.17.1638.34125

2. Leach, J. L., Fortuna, R. B., Jones, B. V. \& Gaskill-Shipley, M. F. (2006). Imaging of Cerebral Venous Thrombosis: Current Techniques, Spectrum of Findings, and Diagnostic Pitfalls. RadioGraphics, 26(suppl_1), S19-S41. doi: doi:10.1148/rg.26si055174

3. Michel, M., Chanet, V., Dechartres, A., Morin, A. -S., Piette, J.-C., Cirasino, L., Emilia, G., Zaja, F., Ruggeri, M., Andrès, E., Bierling, P., Godeau, B. \& Rodeghiero, F. (2009). The spectrum of Evans syndrome in adults: new insight into the disease based on the analysis of 68 cases. Blood, 114(15), 3167-3172.

4. Poon, C. S., Chang, J.-K., Swarnkar, A., Johnson, M. H. \& Wasenko, J. (2007). Radiologic Diagnosis of Cerebral Venous Thrombosis: Pictorial Review. American Journal of Roentgenology, 189(6_supplement), S64-S75. doi: 10.2214/AJR.07.7015

5. Shiozawa, Z., Ueda, R., Mano, T., Tsugane, R. \& Kageyama, N. (1985). Superior sagittal sinus thrombosis associated with Evans' syndrome of haemolytic anaemia. Journal of Neurology, 232 (5), 280-282. doi: 10.1007/BF00313866

6. Yilmaz, S., Oren, H., Irken, G., Turker, M., Yilmaz, E. \& Ada, E. (2005). Cerebral venous thrombosis in a patient with Evans syndrome: a rare association. Ann Hematol, 84(2), 124-126. doi: $10.1007 / \mathrm{s} 00277-004-0963-7$ 